\title{
EXTREMAL CONSTANT SIGN SOLUTIONS AND NODAL SOLUTIONS FOR THE FRACTIONAL $p$-LAPLACIAN
}

\author{
SILVIA FRASSU, ANTONIO IANNIZZOTTO
}

\begin{abstract}
We study a pseudo-differential equation driven by the degenerate fractional $p$-Laplacian, under Dirichlet type conditions in a smooth domain. First we show that the solution set within the order interval given by a sub-supersolution pair is nonempty, directed, and compact, hence endowed with extremal elements. Then, we prove existence of a smallest positive, a biggest negative and a nodal solution, combining variational methods with truncation techniques.
\end{abstract}

Version of November 15, 2019

\section{INTRODUCTION}

In the study of nonlinear boundary value problem, one classical issue is that about the sign of solutions, especially in the case of multiple solutions. Typically, constant sign solutions can be detected as critical points of a truncated energy functional by direct minimization or min-max methods, while the existence of a nodal (i.e., sign-changing) solution is a more delicate question (some classical results, based on Morse theory, can be found in $[1,2,40])$. An interesting approach was proposed in [11] for the Dirichlet problem driven by the Laplacian operator: it consists in proving that the problem admits a smallest positive and a biggest negative solution, plus a third nontrivial solution lying between the two, which must then be nodal. The method used for finding the nodal solution is based on the Fučik spectrum. Such approach was then extended to the $p$-Laplacian in [9], and then combined with a variational characterization of the second eigenvalue to detect a nodal solution under more general assumptions in [16] (see also [20,33] and the monograph [34]).

Recently, many authors have devoted their attention to nonlinear equations driven by nonlocal operators. The present paper is devoted to the study of the following Dirichlet-type problem for a nonlinear fractional equation:

$$
\begin{cases}(-\Delta)_{p}^{s} u=f(x, u) & \text { in } \Omega \\ u=0 & \text { in } \Omega^{c},\end{cases}
$$

where $\Omega \subset \mathbb{R}^{N}(N>1)$ is a bounded domain with $C^{1,1}$ boundary, $p \geqslant 2, s \in(0,1), N>p s$, and $(-\Delta)_{p}^{s}$ denotes the fractional $p$-Laplacian, namely the nonlinear, nonlocal operator defined for all $u: \mathbb{R}^{N} \rightarrow \mathbb{R}$ smooth enough and all $x \in \mathbb{R}^{N}$ by

$$
(-\Delta)_{p}^{s} u(x)=2 \lim _{\varepsilon \rightarrow 0^{+}} \int_{B_{\varepsilon}^{c}(x)} \frac{|u(x)-u(y)|^{p-2}(u(x)-u(y))}{|x-y|^{N+p s}} d y
$$

(which in the linear case $p=2$ reduces to the fractional Laplacian up to a dimensional constant $C(N, p, s)>0$, see $[6,7,14])$. The reaction $f: \Omega \times \mathbb{R} \rightarrow \mathbb{R}$ is a Carathéodory mapping subject to a subcritical growth condition. Problem (1.1) has been intensively studied in the recent literature, both in the semilinear and the nonlinear case. Regarding the semilinear case, we recall the fine regularity results of [36], the existence and multiplicity results obtained for instance in [15,19,23,38], and the study on extremal solutions in [37] (see also the monograph [31]). The nonlinear case is obviously more involved: spectral properties of $(-\Delta)_{p}^{s}$ were studied in $[4,17,18,21,30]$, a detailed regularity theory was developed in $[3,24,25,28,29]$ (some results about Sobolev and Hölder regularity being only proved for the degenerate case $p>2$ ), maximum and comparison principles have appeared in [12,27], while existence and multiplicity of solutions have been obtained for instance in $[10,13,18,22,39]$ (see also the surveys [32,35]). For the purposes of the present study, we recall in particular [26], where it was proved that the local minimizers of the energy functional corresponding to problem (1.1) in the

2010 Mathematics Subject Classification. 35A15, 35R11, 58E05.

Key words and phrases. Fractional $p$-Laplacian, extremal constant sign solutions, nodal solutions, critical point theory. 
topologies of $W_{0}^{s, p}(\Omega)$ and of the weighted Hölder space $C_{s}^{0}(\bar{\Omega})$, respectively, coincide (namely, a nonlinear fractional analogue of the classical result of [5]).

Here we focus on the structure of the set $\mathcal{S}(\underline{u}, \bar{u})$, namely the set of solutions of (1.1) lying within the interval $[\underline{u}, \bar{u}]$ where $\underline{u}$ and $\bar{u}$ are a subsolution and a supersolution of (1.1), respectively, with $\underline{u} \leqslant \bar{u}$ in $\Omega$. We shall prove that $\mathcal{S}(\underline{u}, \bar{u})$ is nonempty, directed, and compact in $W_{0}^{s, p}(\Omega)$, hence endowed with extremal elements.

Then, we will assume that $f(x, \cdot)$ is $(p-1)$-sublinear at infinity and asymptotically linear near the origin without resonance on the first eigenvalue, and prove that (1.1) has a smallest positive solution $u_{+}$and a biggest negative solution $u_{-}$. Finally, under more restrictive assumptions on the behavior of $f(x, \cdot)$ near the origin, we will prove existence of a nodal solution $\tilde{u}$ s.t. $u_{-} \leqslant \tilde{u} \leqslant u_{+}$in $\Omega$, thus extending some results of $[9,16]$ to the fractional $p$-Laplacian.

We remark that our results are new (to our knowledge) even in the semilinear case $p=2$, and that the structure of the set $\mathcal{S}(\underline{u}, \bar{u})$ can provide valuable information about extremal solutions also in different frameworks. The paper has the following structure: in Section 2 we collect the necessary preliminaries; in Section 3 we study the properties of the solution set; in Section 4 we show existence of extremal constant sign solutions; and in Section 5 we prove existence of a nontrivial nodal solution.

Notation: Throughout the paper, for any $A \subset \mathbb{R}^{N}$ we shall set $A^{c}=\mathbb{R}^{N} \backslash A$. For any two measurable functions $f, g: \Omega \rightarrow \mathbb{R}, f \leqslant g$ will mean that $f(x) \leqslant g(x)$ for a.e. $x \in \Omega$ (and similar expressions). The positive (resp., negative) part of $f$ is denoted $f^{+}$(resp., $f^{-}$). If $X$ is an ordered Banach space, then $X_{+}$will denote its non-negative order cone. For all $r \in[1, \infty],\|\cdot\|_{r}$ denotes the standard norm of $L^{r}(\Omega)$ (or $L^{r}\left(\mathbb{R}^{N}\right)$, which will be clear from the context). Every function $u$ defined in $\Omega$ will be identified with its 0 -extension to $\mathbb{R}^{N}$. Moreover, $C$ will denote a positive constant (whose value may change case by case).

\section{Preliminaries}

In this section we collect some useful results related to the fractional $p$-Laplacian. First we fix a functionalanalytical framework, following [14,22]. First, for all measurable $u: \mathbb{R}^{N} \rightarrow \mathbb{R}$ we set

$$
[u]_{s, p}^{p}=\iint_{\mathbb{R}^{N} \times \mathbb{R}^{N}} \frac{|u(x)-u(y)|^{p}}{|x-y|^{N+p s}} d \mu,
$$

where $d \mu=|x-y|^{-N-p s} d x d y$. Then we define the following fractional Sobolev spaces:

$$
\begin{gathered}
W^{s, p}\left(\mathbb{R}^{N}\right)=\left\{u \in L^{p}\left(\mathbb{R}^{N}\right):[u]_{s, p}<\infty\right\}, \\
W_{0}^{s, p}(\Omega)=\left\{u \in W^{s, p}\left(\mathbb{R}^{N}\right): u(x)=0 \text { in } \Omega^{c}\right\},
\end{gathered}
$$

the latter being a uniformly convex, separable Banach space with norm $\|u\|_{s, p}=[u]_{s, p}$ and dual $W^{-s, p^{\prime}}(\Omega)$ (with norm $\|\cdot\|_{-s, p^{\prime}}$ ). Set $p_{s}^{*}=N p /\left(N-p s\right.$ ), then the embedding $W_{0}^{s, p}(\Omega) \hookrightarrow L^{q}(\Omega)$ is continuous for all $q \in\left[1, p_{s}^{*}\right]$ and compact for all $q \in\left[1, p_{s}^{*}\right)$, with embedding constant $c_{q}>0$.

We denote $\widetilde{W}^{s, p}(\Omega)$ the space of all $u \in L_{\mathrm{loc}}^{p}\left(\mathbb{R}^{N}\right)$ s.t. $u \in W^{s, p}(U)$ for some open $U \subseteq \mathbb{R}^{N}, \bar{\Omega} \subset U$, and

$$
\int_{\mathbb{R}^{N}} \frac{|u(x)|^{p-1}}{(1+|x|)^{N+p s}} d x<\infty .
$$

Clearly, $W_{0}^{s, p}(\Omega) \subset \widetilde{W}^{s, p}(\Omega)$. By [24, Lemma 2.3], for any $u \in \widetilde{W}^{s, p}(\Omega)$ we can define $(-\Delta)_{p}^{s} u \in W^{-s, p^{\prime}}(\Omega)$ by setting for all $v \in W_{0}^{s, p}(\Omega)$

$$
\left\langle(-\Delta)_{p}^{s} u, v\right\rangle=\iint_{\mathbb{R}^{N} \times \mathbb{R}^{N}}|u(x)-u(y)|^{p-1}(u(x)-u(y))(v(x)-v(y)) d \mu .
$$

The definition above agrees with (1.2) when $u$ lies in the Schwartz space of $C^{\infty}$, rapidly decaying functions in $\mathbb{R}^{N}$. In the next lemma we recall some useful properties of $(-\Delta)_{p}^{s}$ in $W_{0}^{s, p}(\Omega)$ :

Lemma 2.1. $(-\Delta)_{p}^{s}: W_{0}^{s, p}(\Omega) \rightarrow W^{-s, p^{\prime}}(\Omega)$ is a monotone, continuous, $(S)_{+}$-operator.

Proof. By [26, Lemma 2.3] (with $q=1$ ) we have for all $u, v \in W_{0}^{s, p}(\Omega)$

$$
\left\langle(-\Delta)_{p}^{s} u-(-\Delta)_{p}^{s} v, u-v\right\rangle \geqslant 0
$$


hence $(-\Delta)_{p}^{s}$ is monotone. Plus, $(-\Delta)_{p}^{s}$ is continuous as the Gâteaux derivative of the $C^{1}$-functional $u \mapsto$ $\frac{\|u\|_{s, p}^{p}}{p}$. Finally, if $u_{n} \rightarrow u$ in $W_{0}^{s, p}(\Omega)$ and

$$
\limsup _{n}\left\langle(-\Delta)_{p}^{s} u_{n}, u_{n}-u\right\rangle \leqslant 0
$$

then for all $n \in \mathbb{N}$ we have

$$
\begin{aligned}
& \left(\left\|u_{n}\right\|_{s, p}^{p-1}-\left\|u_{n}\right\|_{s, p}^{p-1}\right)\left(\|u\|_{s, p}-\|u\|_{s, p}\right)=\left\|u_{n}\right\|_{s, p}^{p}-\left\|u_{n}\right\|_{s, p}^{p-1}\|u\|_{s, p}-\left\|u_{n}\right\|_{s, p}\|u\|_{s, p}^{p-1}+\|u\|_{s, p}^{p} \\
& \leq\left\langle(-\Delta)_{p}^{s} u_{n}, u_{n}\right\rangle-\left\langle(-\Delta)_{p}^{s} u_{n}, u\right\rangle-\left\langle(-\Delta)_{p}^{s} u, u_{n}\right\rangle+\left\langle(-\Delta)_{p}^{s} u, u\right\rangle \\
& =\left\langle(-\Delta)_{p}^{s} u_{n}, u_{n}-u\right\rangle+\left\langle(-\Delta)_{p}^{s} u, u-u_{n}\right\rangle \leq \mathbf{o}(1),
\end{aligned}
$$

hence $\left\|u_{n}\right\|_{s, p} \rightarrow\|u\|_{s, p}$. By uniform convexity of $W_{0}^{s, p}(\Omega), u_{n} \rightarrow u$ in $W_{0}^{s, p}(\Omega)$. Therefore, $(-\Delta)_{p}^{s}$ is an $(S)_{+}$-operator.

Now we introduce basic hypothesis on the reaction $f$ :

$\mathbf{H}_{0} f: \Omega \times \mathbb{R} \rightarrow \mathbb{R}$ is a Carathéodory function s.t. for a.e. $x \in \Omega$ and all $t \in \mathbb{R}$

$$
|f(x, t)| \leq c_{0}\left(1+|t|^{q-1}\right) \quad\left(c_{0}>0, q \in\left(p, p_{s}^{*}\right)\right)
$$

We recall some definitions:

Definition 2.2. Let $u \in \widetilde{W}^{s, p}(\Omega)$ :

(i) $u$ is a supersolution of (1.1) if $u \geqslant 0$ in $\Omega^{c}$ and for all $v \in W_{0}^{s, p}(\Omega)_{+}$

$$
\left\langle(-\Delta)_{p}^{s} u, v\right\rangle \geqslant \int_{\Omega} f(x, u) v d x
$$

(ii) $u$ is a subsolution of (1.1) if $u \leqslant 0$ in $\Omega^{c}$ and for all $v \in W_{0}^{s, p}(\Omega)_{+}$

$$
\left\langle(-\Delta)_{p}^{s} u, v\right\rangle \leqslant \int_{\Omega} f(x, u) v d x .
$$

We say that $(\underline{u}, \bar{u}) \in \widetilde{W}^{s, p}(\Omega) \times \widetilde{W}^{s, p}(\Omega)$ is a sub-supersolution pair of (1.1), if $\underline{u}$ is a subsolution, $\bar{u}$ is a supersolution, and $\underline{u} \leqslant \bar{u}$ in $\Omega$.

Definition 2.3. $u \in W_{0}^{s, p}(\Omega)$ is a solution of (1.1) if for all $v \in W_{0}^{s, p}(\Omega)$

$$
\left\langle(-\Delta)_{p}^{s} u, v\right\rangle=\int_{\Omega} f(x, u) v d x .
$$

Clearly, $u \in W_{0}^{s, p}(\Omega)$ is a solution of (1.1) iff it is both a supersolution and a subsolution. Sub-, supersolutions, and solutions of similar problems will be meant in the same sense as in Definitions 2.2, 2.3 above.

We will need the following a priori bound for solutions of (1.1):

Lemma 2.4. [26, Lemma 2.1] Let $\mathbf{H}_{0}$ hold, $u \in W_{0}^{s, p}(\Omega)$ be a solution of (1.1). Then, $u \in L^{\infty}(\Omega)$ with $\|u\|_{\infty} \leqslant C$, for some $C=C\left(\|u\|_{s, p}\right)>0$.

We define weighted Hölder-type spaces with weight $\mathrm{d}_{\Omega}^{s}(x)=\operatorname{dist}\left(x, \Omega^{c}\right)^{s}$, along with their norms:

$$
C_{s}^{0}(\bar{\Omega})=\left\{u \in C^{0}(\bar{\Omega}): \frac{u}{\mathrm{~d}_{\Omega}^{s}} \in C^{0}(\bar{\Omega})\right\}, \quad\|u\|_{0, s}=\left\|\frac{u}{\mathrm{~d}_{\Omega}^{s}}\right\|_{\infty},
$$

and for all $\alpha \in(0,1)$

$$
C_{s}^{\alpha}(\bar{\Omega})=\left\{u \in C^{0}(\bar{\Omega}): \frac{u}{\mathrm{~d}_{\Omega}^{s}} \in C^{\alpha}(\bar{\Omega})\right\}, \quad\|u\|_{\alpha, s}=\|u\|_{0, s}+\sup _{x \neq y} \frac{\left|u(x) / \mathrm{d}_{\Omega}^{s}(x)-u(y) / \mathrm{d}_{\Omega}^{s}(y)\right|}{|x-y|^{\alpha}} .
$$

The embedding $C_{s}^{\alpha}(\bar{\Omega}) \hookrightarrow C_{s}^{0}(\bar{\Omega})$ is compact for all $\alpha \in(0,1)$. Unlike in $W_{0}^{s, p}(\Omega)$, the positive cone $C_{s}^{0}(\bar{\Omega})_{+}$ of $C_{s}^{0}(\bar{\Omega})$ has a nonempty interior given by

$$
\operatorname{int}\left(C_{s}^{0}(\bar{\Omega})_{+}\right)=\left\{u \in C_{s}^{0}(\bar{\Omega}): \frac{u(x)}{\mathrm{d}_{\Omega}^{s}(x)}>0 \text { in } \bar{\Omega}\right\}
$$


(see [22, Lemma 5.1]). Consider the following Dirichlet problem, with right-hand side $g \in L^{\infty}(\Omega)$ :

$$
\begin{cases}(-\Delta)_{p}^{s} u=g(x) & \text { in } \Omega \\ u=0 & \text { in } \Omega^{c} .\end{cases}
$$

We have the following regularity result:

Lemma 2.5. [25, Theorem 1.1] Let $g \in L^{\infty}(\Omega), u \in W_{0}^{s, p}(\Omega)$ be a solution of $(2.2)$. Then, $u \in C_{s}^{\alpha}(\bar{\Omega})$ with $\|u\|_{\alpha, s} \leqslant C\|g\|_{\infty}^{\frac{1}{p-1}}$, for some $\alpha \in(0, s], C=C(\Omega)>0$.

Combining Lemmas 2.4, 2.5 we see that any solution of (1.1) under $\mathbf{H}_{0}$ lies in $C_{s}^{\alpha}(\bar{\Omega})$, with a uniform estimate on the $C_{s}^{\alpha}(\bar{\Omega})$-norm. In the final part of our study, we will follow a variational approach. We define an energy functional for problem (1.1) by setting for all $(x, t) \in \Omega \times \mathbb{R}$

$$
F(x, t)=\int_{0}^{t} f(x, \tau) d \tau,
$$

and for all $u \in W_{0}^{s, p}(\Omega)$

$$
\Phi(u)=\frac{\|u\|_{s, p}^{p}}{p}-\int_{\Omega} F(x, u) d x .
$$

By $\mathbf{H}_{0}$, it is easily seen that $\Phi \in C^{1}\left(W_{0}^{s, p}(\Omega)\right)$ and the solutions of (1.1) coincide with the critical points of $\Phi$. We will need the following equivalence result for local minimizers of $\Phi$ in $W_{0}^{s, p}(\Omega)$ and in $C_{s}^{0}(\bar{\Omega})$ :

Lemma 2.6. [26, Theorem 1.1] Let $\mathbf{H}_{0}$ hold, $u \in W_{0}^{s, p}(\Omega)$. Then, the following are equivalent:

(i) there exists $\rho>0$ s.t. $\Phi(u+v) \geqslant \Phi(u)$ for all $v \in W_{0}^{s, p}(\Omega),\|v\|_{s, p} \leqslant \rho$;

(ii) there exists $\sigma>0$ s.t. $\Phi(u+v) \geqslant \Phi(u)$ for all $v \in W_{0}^{s, p}(\Omega) \cap C_{s}^{0}(\bar{\Omega}),\|v\|_{0, s} \leqslant \sigma$.

Since we are mainly interested in constant sign solutions, we will need a strong maximum principle and Hopf's lemma. Consider the problem

$$
\begin{cases}(-\Delta)_{p}^{s} u=-c(x)|u|^{p-2} u & \text { in } \Omega \\ u=0 & \text { in } \Omega^{c},\end{cases}
$$

with $c \in C^{0}(\bar{\Omega})_{+}$. Then we have the following:

Lemma 2.7. [12, Theorem 1.5] Let $c \in C^{0}(\bar{\Omega})_{+}, u \in \widetilde{W}^{s, p}(\Omega)_{+} \backslash\{0\}$ be a supersolution of (2.3). Then, $u>0$ in $\Omega$ and for any $x_{0} \in \partial \Omega$

$$
\liminf _{\Omega \ni x \rightarrow x_{0}} \frac{u(x)}{\mathrm{d}_{\Omega}^{s}(x)}>0 .
$$

Finally, we recall some spectral properties of $(-\Delta)_{p}^{s}$ (see $[13,21]$ and $\left[18\right.$, Proposition 3.4]). Let $\rho \in L^{\infty}(\Omega)_{+} \backslash$ $\{0\}$ and consider the following weighted eigenvalue problem:

$$
\begin{cases}(-\Delta)_{p}^{s} u=\lambda \rho(x)|u|^{p-2} u & \text { in } \Omega \\ u=0 & \text { on } \Omega^{c} .\end{cases}
$$

Lemma 2.8. Let $\rho \in L^{\infty}(\Omega)_{+} \backslash\{0\}$. Then, (2.4) has an unbounded sequence of variational eigenvalues

$$
0<\lambda_{1}(\rho)<\lambda_{2}(\rho) \leqslant \ldots \leqslant \lambda_{k}(\rho) \leqslant \ldots
$$

The first eigenvalue admits the following variational characterization:

$$
\lambda_{1}(\rho)=\inf _{u \in W_{0}^{s, p}(\Omega) \backslash\{0\}} \frac{\|u\|_{s, p}^{p}}{\int_{\Omega} \rho(x)|u|^{p} d x},
$$

and

(i) $\lambda_{1}(\rho)>0$ is simple, isolated and attained at an unique positive eigenfunction $\hat{u}_{1}(\rho) \in W_{0}^{s, p}(\Omega) \cap$ $\operatorname{int}\left(C_{s}^{0}(\bar{\Omega})_{+}\right)$s.t. $\int_{\Omega} \rho(x)\left|\hat{u}_{1}\right|^{p} d x=1$;

(ii) if $u \in W_{0}^{s, p}(\Omega) \backslash\{0\}$ is an eigenfunction of (2.4) associated to any eigenvalue $\lambda>\lambda_{1}(\rho)$, then $u$ is nodal;

(iii) if $\tilde{\rho} \in L^{\infty}(\Omega)_{+} \backslash\{0\}$ is s.t. $\tilde{\rho} \leqslant \rho, \tilde{\rho} \not \equiv \rho$, then $\lambda_{1}(\rho)<\lambda_{1}(\tilde{\rho})$. 
When $\rho \equiv 1$ we set $\lambda_{1}(\rho)=\lambda_{1}$ and $\hat{u}_{1}(\rho)=\hat{u}_{1}$. Moreover, the second (non-weighted) eigenvalue admits the following variational characterization:

$$
\lambda_{2}=\inf _{\gamma \in \Gamma_{1}} \max _{t \in[0,1]}\|\gamma(t)\|_{s, p}^{p}
$$

where

$$
\Gamma_{1}=\left\{\gamma \in C\left([0,1], W_{0}^{s, p}(\Omega)\right): \gamma(0)=\hat{u}_{1}, \gamma(1)=-\hat{u}_{1},\|\gamma(t)\|_{p}=1 \text { for all } t \in[0,1]\right\},
$$

see $[4$, Theorem 5.3].

\section{Solutions in a SUB-SUPERSOlUtion INTERVAL}

In this section we consider a sub-supersolution pair $(\underline{u}, \bar{u})$ and study the set

$$
\mathcal{S}(\underline{u}, \bar{u})=\left\{u \in W_{0}^{s, p}(\Omega): u \text { solves }(1.1), \underline{u} \leqslant u \leqslant \bar{u}\right\} .
$$

On spaces $W_{0}^{s, p}(\Omega), \widetilde{W}^{s, p}(\Omega)$ we consider the partial pointwise order, inducing a lattice structure. We set $u \wedge v=\min \{u, v\}$ and $u \vee v=\max \{u, v\}$.

The first result shows that the pointwise minimum of supersolutions is a supersolution, as well as the maximum of subsolutions is a subsolution (we give the proof in full detail, as it requires some careful calculations):

Lemma 3.1. Let $\mathbf{H}_{0}$ hold and $u_{1}, u_{2} \in \widetilde{W}^{s, p}(\Omega)$ :

(i) if $u_{1}, u_{2}$ are supersolutions of (1.1), then so is $u_{1} \wedge u_{2}$;

(ii) if $u_{1}, u_{2}$ are subsolutions of (1.1) then so is $u_{1} \vee u_{2}$.

Proof. We prove $(i)$. We have for $i=1,2$

$$
\begin{cases}\left\langle(-\Delta)_{p}^{s} u_{i}, v\right\rangle \geqslant \int_{\Omega} f\left(x, u_{i}\right) v d x & \text { for all } v \in W_{0}^{s, p}(\Omega)_{+} \\ u_{i} \geqslant 0 & \text { in } \Omega^{c} .\end{cases}
$$

Set $u=u_{1} \wedge u_{2} \in \widetilde{W}^{s, p}(\Omega)$ (by the lattice structure of $\widetilde{W}^{s, p}(\Omega)$ ), then $u \geqslant 0$ in $\Omega^{c}$. Set also

$$
A_{1}=\left\{x \in \mathbb{R}^{N}: u_{1}(x)<u_{2}(x)\right\}, \quad A_{2}=A_{1}^{c} .
$$

Now fix $\varphi \in C_{c}^{\infty}(\Omega)_{+}, \varepsilon>0$, and set for all $t \in \mathbb{R}$

$$
\tau_{\varepsilon}(t)= \begin{cases}0 & \text { if } t \leqslant 0 \\ \frac{t}{\varepsilon} & \text { if } 0<t<\varepsilon \\ 1 & \text { if } t \geqslant \varepsilon\end{cases}
$$

The mapping $\tau_{\varepsilon}: \mathbb{R} \rightarrow \mathbb{R}$ is Lipschitz continuous, nondecreasing, and $0 \leqslant \tau_{\varepsilon}(t) \leqslant 1$ for all $t \in \mathbb{R}$, and clearly

$$
\tau_{\varepsilon}\left(u_{2}-u_{1}\right) \rightarrow \chi_{A_{1}}, \quad 1-\tau_{\varepsilon}\left(u_{2}-u_{1}\right) \rightarrow \chi_{A_{2}}
$$

a.e. in $\mathbb{R}^{N}$, as $\varepsilon \rightarrow 0^{+}$, with dominated convergence. Testing (3.1) with $\tau_{\varepsilon}\left(u_{2}-u_{1}\right) \varphi,\left(1-\tau_{\varepsilon}\left(u_{2}-u_{1}\right)\right) \varphi \in$ $W_{0}^{s, p}(\Omega)_{+}$for $i=1,2$ respectively, we get

$$
\begin{aligned}
& \left\langle(-\Delta)_{p}^{s} u_{1}, \tau_{\varepsilon}\left(u_{2}-u_{1}\right) \varphi\right\rangle+\left\langle(-\Delta)_{p}^{s} u_{2},\left(1-\tau_{\varepsilon}\left(u_{2}-u_{1}\right)\right) \varphi\right\rangle \\
& \geqslant \int_{\Omega} f\left(x, u_{1}\right) \tau_{\varepsilon}\left(u_{2}-u_{1}\right) \varphi d x+\int_{\Omega} f\left(x, u_{2}\right)\left(1-\tau_{\varepsilon}\left(u_{2}-u_{1}\right)\right) \varphi d x
\end{aligned}
$$

We focus on the left-hand side of (3.2). Setting for brevity $\tau_{\varepsilon}=\tau_{\varepsilon}\left(u_{2}-u_{1}\right)$ and $a^{p-1}=|a|^{p-2} a$ for all $a \in \mathbb{R}$, and recalling that $\tau_{\varepsilon}=0$ in $A_{2}$, while $\tau_{\varepsilon} \rightarrow 1$ in $A_{1}$ as $\varepsilon \rightarrow 0^{+}$, we get

$$
\begin{aligned}
& \left\langle(-\Delta)_{p}^{s} u_{1}, \tau_{\varepsilon} \varphi\right\rangle+\left\langle(-\Delta)_{p}^{s} u_{2},\left(1-\tau_{\varepsilon}\right) \varphi\right\rangle \\
& =\iint_{\mathbb{R}^{N} \times \mathbb{R}^{N}}\left(u_{1}(x)-u_{1}(y)\right)^{p-1}\left(\tau_{\varepsilon}(x) \varphi(x)-\tau_{\varepsilon}(y) \varphi(y)\right) d \mu \\
& +\iint_{\mathbb{R}^{N} \times \mathbb{R}^{N}}\left(u_{2}(x)-u_{2}(y)\right)^{p-1}\left[\left(1-\tau_{\varepsilon}(x)\right) \varphi(x)-\left(1-\tau_{\varepsilon}(y)\right) \varphi(y)\right] d \mu \\
& =: I .
\end{aligned}
$$


Using the definition of $A_{1}$ and $A_{2}$, we obtain

$$
\begin{aligned}
I & =\iint_{A_{1} \times A_{1}}\left(u_{1}(x)-u_{1}(y)\right)^{p-1}(\varphi(x)-\varphi(y)) \tau_{\varepsilon}(x) d \mu \\
& +\iint_{A_{1} \times A_{1}}\left(u_{1}(x)-u_{1}(y)\right)^{p-1} \varphi(y)\left(\tau_{\varepsilon}(x)-\tau_{\varepsilon}(y)\right) d \mu \\
& +\iint_{A_{1} \times A_{2}}\left(u_{1}(x)-u_{1}(y)\right)^{p-1} \varphi(x) \tau_{\varepsilon}(x) d \mu \\
& -\iint_{A_{2} \times A_{1}}\left(u_{1}(x)-u_{1}(y)\right)^{p-1} \varphi(y) \tau_{\varepsilon}(y) d \mu \\
& +\iint_{A_{1} \times A_{1}}\left(u_{2}(x)-u_{2}(y)\right)^{p-1}(\varphi(x)-\varphi(y))\left(1-\tau_{\varepsilon}(x)\right) d \mu \\
& -\iint_{A_{1} \times A_{1}}\left(u_{2}(x)-u_{2}(y)\right)^{p-1} \varphi(y)\left(\tau_{\varepsilon}(x)-\tau_{\varepsilon}(y)\right) d \mu \\
& +\iint_{A_{1} \times A_{2}}\left(u_{2}(x)-u_{2}(y)\right)^{p-1}(\varphi(x)-\varphi(y))\left(1-\tau_{\varepsilon}(x)\right) d \mu \\
& -\iint_{A_{1} \times A_{2}}\left(u_{2}(x)-u_{2}(y)\right)^{p-1} \varphi(y) \tau_{\varepsilon}(x) d \mu \\
& +\iint_{A_{2} \times A_{1}}\left(u_{2}(x)-u_{2}(y)\right)^{p-1} \varphi(x) \tau_{\varepsilon}(y) d \mu \\
& +\iint_{A_{2} \times A_{1}}\left(u_{2}(x)-u_{2}(y)\right)^{p-1}(\varphi(x)-\varphi(y))\left(1-\tau_{\varepsilon}(y)\right) d \mu \\
& +\iint_{A_{2} \times A_{2}}\left(u_{2}(x)-u_{2}(y)\right)^{p-1}(\varphi(x)-\varphi(y)) d \mu .
\end{aligned}
$$

We then put together the integrals with the same letter and note that (E), (F), (G) $\rightarrow 0$ as $\varepsilon \rightarrow 0^{+}$. So, we have

$$
\begin{aligned}
I & =\iint_{A_{1} \times A_{1}}\left(u_{1}(x)-u_{1}(y)\right)^{p-1}(\varphi(x)-\varphi(y)) d \mu \\
& +\iint_{A_{1} \times A_{1}}\left[\left(u_{1}(x)-u_{1}(y)\right)^{p-1}-\left(u_{2}(x)-u_{2}(y)\right)^{p-1}\right] \varphi(y)\left(\tau_{\varepsilon}(x)-\tau_{\varepsilon}(y)\right) d \mu \\
& +\iint_{A_{1} \times A_{2}}\left[\left(u_{1}(x)-u_{1}(y)\right)^{p-1} \varphi(x)-\left(u_{2}(x)-u_{2}(y)\right)^{p-1} \varphi(y)\right] \tau_{\varepsilon}(x) d \mu \\
& +\iint_{A_{2} \times A_{1}}\left[\left(u_{2}(x)-u_{2}(y)\right)^{p-1} \varphi(x)-\left(u_{1}(x)-u_{1}(y)\right)^{p-1} \varphi(y)\right] \tau_{\varepsilon}(y) d \mu \\
& +\iint_{A_{2} \times A_{2}}\left(u_{2}(x)-u_{2}(y)\right)^{p-1}(\varphi(x)-\varphi(y)) d \mu \\
& +\mathbf{o}(1) .
\end{aligned}
$$

Now we note that for all $x, y \in A_{1}$

$$
u_{1}(x)-u_{1}(y) \geqslant u_{2}(x)-u_{2}(y) \Leftrightarrow u_{2}(y)-u_{1}(y) \geqslant u_{2}(x)-u_{1}(x) \Leftrightarrow \tau_{\varepsilon}(y) \geqslant \tau_{\varepsilon}(x),
$$

hence the integrand in (B) is negative. Besides, for all $x \in A_{1}, y \in A_{2}$

$$
u_{1}(x)-u_{1}(y) \leqslant u_{1}(x)-u_{2}(y) \leqslant u_{2}(x)-u_{2}(y)
$$

and for all $x \in A_{2}, y \in A_{1}$

$$
u_{2}(x)-u_{2}(y) \leqslant u_{2}(x)-u_{1}(y) \leqslant u_{1}(x)-u_{1}(y)
$$


so we can estimate the integrands in (C), (D) respectively and get

$$
\begin{aligned}
I & \leqslant \iint_{A_{1} \times A_{1}}\left(u_{1}(x)-u_{1}(y)\right)^{p-1}(\varphi(x)-\varphi(y)) d \mu \\
& +\iint_{A_{1} \times A_{2}}\left(u_{1}(x)-u_{2}(y)\right)^{p-1}(\varphi(x)-\varphi(y)) d \mu \\
& +\iint_{A_{2} \times A_{1}}\left[\left(u_{2}(x)-u_{1}(y)\right)^{p-1}(\varphi(x)-\varphi(y)) d \mu\right. \\
& +\iint_{A_{2} \times A_{2}}\left(u_{2}(x)-u_{2}(y)\right)^{p-1}(\varphi(x)-\varphi(y)) d \mu+\mathbf{o}(1) \\
& =\left\langle(-\Delta)_{p}^{s} u, \varphi\right\rangle+\mathbf{o}(1) .
\end{aligned}
$$

All in all, we have

$$
\left\langle(-\Delta)_{p}^{s} u_{1}, \tau_{\varepsilon}\left(u_{2}-u_{1}\right) \varphi\right\rangle+\left\langle(-\Delta)_{p}^{s} u_{2},\left(1-\tau_{\varepsilon}\left(u_{2}-u_{1}\right)\right) \varphi\right\rangle\left\langle(-\Delta)_{p}^{s} u, \varphi\right\rangle+\mathbf{o}(1),
$$

as $\varepsilon \rightarrow 0^{+}$. Regarding the right-hand side of (3.2), we use the bounds from $\mathbf{H}_{0}$ and the definition of $\tau_{\varepsilon}$ to get

$$
\begin{gathered}
\left|f\left(\cdot, u_{1}\right) \tau_{\varepsilon}^{+}\left(u_{2}-u_{1}\right) \varphi\right| \leqslant c_{0}\left(1+\left|u_{1}\right|^{q-1}\right) \varphi \\
\left|f\left(\cdot, u_{2}\right)\left(1-\tau_{\varepsilon}^{+}\left(u_{2}-u_{1}\right)\right) \varphi\right| \leqslant c_{0}\left(1+\left|u_{2}\right|^{q-1}\right) \varphi,
\end{gathered}
$$

and pass to the limit as $\varepsilon \rightarrow 0^{+}$:

$$
\begin{aligned}
& \int_{\Omega} f\left(x, u_{1}\right) \tau_{\varepsilon}\left(u_{2}-u_{1}\right) \varphi d x+\int_{\Omega} f\left(x, u_{2}\right)\left(1-\tau_{\varepsilon}\left(u_{2}-u_{1}\right)\right) \varphi d x \\
& =\int_{\Omega} f\left(x, u_{1}\right) \chi_{A_{1}} \varphi d x+\int_{\Omega} f\left(x, u_{2}\right) \chi_{A_{2}} \varphi d x+\mathbf{o}(1) \\
& =\int_{\Omega} f(x, u) \varphi d x+\mathbf{o}(1) .
\end{aligned}
$$

Plugging (3.3), (3.4) into (3.2) we have for all $\varphi \in C_{c}^{\infty}(\bar{\Omega})_{+}$

$$
\left\langle(-\Delta)_{p}^{s} u, \varphi\right\rangle \geqslant \int_{\Omega} f(x, u) \varphi d x
$$

By density, the same holds with test functions in $W_{0}^{s, p}(\Omega)_{+}$, hence $u$ is a supersolution of (1.1), which proves (i). Similarly we prove $(i i)$.

Now we consider a sub-supersolution pair $(\underline{u}, \bar{u})$ and we study the set $\mathcal{S}(\underline{u}, \bar{u})$. We begin with a subsupersolution principle, showing that $\mathcal{S}(\underline{u}, \bar{u}) \neq \emptyset$ :

Lemma 3.2. Let $\mathbf{H}_{0}$ hold and $(\underline{u}, \bar{u})$ be a sub-supersolution pair of (1.1). Then, there exists $u \in \mathcal{S}(\underline{u}, \bar{u})$.

Proof. In this argument we use some nonlinear operator theory from [8]. First we define $A=(-\Delta)_{p}^{s}$ : $W_{0}^{s, p}(\Omega) \rightarrow W^{-s, p^{\prime}}(\Omega)$. By Lemma $2.1 A$ is monotone and continuous, hence hemicontinuous [8, Definition $2.95($ iii $)$ ], therefore $A$ is pseudomonotone [8, Lemma $2.98(i)$.

Besides, we set for all $(x, t) \in \Omega \times \mathbb{R}$

$$
\tilde{f}(x, t)= \begin{cases}f(x, \underline{u}(x)) & \text { if } t \leqslant \underline{u}(x) \\ f(x, t) & \text { if } \underline{u}(x)<t<\bar{u}(x) \\ f(x, \bar{u}(x)) & \text { if } t \geqslant \bar{u}(x) .\end{cases}
$$

In general, $\tilde{f}$ does not satisfy $\mathbf{H}_{0}$, but still $\tilde{f}: \Omega \times \mathbb{R} \rightarrow \mathbb{R}$ is a Carathéodory function s.t. for a.e. $x \in \Omega$ and all $t \in \mathbb{R}$

$$
|\tilde{f}(x, t)| \leqslant c_{0}\left(1+|\underline{u}|^{q-1}+|\bar{u}|^{q-1}\right) .
$$

We define $B: W_{0}^{s, p}(\Omega) \rightarrow W^{-s, p^{\prime}}(\Omega)$ by setting for all $u, v \in W_{0}^{s, p}(\Omega)$

$$
\langle B(u), v\rangle=-\int_{\Omega} \tilde{f}(x, u) v d x,
$$


well posed by (3.5), as $|\underline{u}|^{q-1},|\bar{u}|^{q-1} \in L^{q^{\prime}}(\Omega)$. We prove that $B$ is strongly continuous [8, Definition 2.95 $(i v)$ ]. Indeed, let $\left(u_{n}\right)$ be a sequence s.t. $u_{n} \rightarrow u$ in $W_{0}^{s, p}(\Omega)$, passing to a subsequence if necessary, we have $u_{n} \rightarrow u$ in $L^{q}(\Omega), u_{n}(x) \rightarrow u(x)$ and $\left|u_{n}(x)\right| \leqslant h(x)$ for a.e. $x \in \Omega$, for some $h \in L^{q}(\Omega)$. Therefore, for all $n \in \mathbb{N}$, by (3.5) we have for a.e. $x \in \Omega$

$$
\left|\tilde{f}\left(x, u_{n}\right)-\tilde{f}(x, u)\right| \leqslant 2 c_{0}\left(1+|\underline{u}|^{q-1}+|\bar{u}|^{q-1}\right) \in L^{q^{\prime}}(\Omega),
$$

while by continuity of $f(x, \cdot)$ we have $\tilde{f}\left(x, u_{n}\right) \rightarrow \tilde{f}(x, u)$. Hence, for all $v \in W_{0}^{s, p}(\Omega)$,

$$
\begin{aligned}
\left|\left\langle B\left(u_{n}\right)-B(u), v\right\rangle\right| & \leqslant \int_{\Omega}\left|\tilde{f}\left(x, u_{n}\right)-\tilde{f}(x, u) \| v\right| d x \\
& \leqslant\left\|\tilde{f}\left(\cdot, u_{n}\right)-\tilde{f}(\cdot, u)\right\|_{q^{\prime}}\|v\|_{q}
\end{aligned}
$$

and the latter tends to 0 as $n \rightarrow \infty$, uniformly with respect to $v$. Therefore $B\left(u_{n}\right) \rightarrow B(u)$ in $W^{-s, p^{\prime}}(\Omega)$. By $[8$, Lemma $2.98(i i)], B$ is pseudomonotone. Thus, $A+B$ is pseudomonotone.

Now we prove that $A+B$ is bounded. Indeed, for all $u \in W_{0}^{s, p}(\Omega)$ we have $\|A(u)\|_{-s, p^{\prime}} \leqslant\|u\|_{s, p}^{p-1}$ and

$$
\begin{aligned}
\|B(u)\|_{-s, p^{\prime}} & =\sup _{\|v\|_{s, p} \leqslant 1} \int_{\Omega} \tilde{f}(x, u) v d x \\
& \leqslant C\|\tilde{f}(\cdot, u)\|_{q^{\prime}} \\
& \leqslant C\left(1+\|\underline{u}\|_{q}^{q-1}+\|\bar{u}\|_{q}^{q-1}\right),
\end{aligned}
$$

where we have used (3.5) and the continuous embedding $W_{0}^{s, p}(\Omega) \hookrightarrow L^{q}(\Omega)$.

Finally we prove that $A+B$ is coercive. Indeed, for all $u \in W_{0}^{s, p}(\Omega) \backslash\{0\}$ we have

$$
\begin{aligned}
\frac{\langle A(u)+B(u), u\rangle}{\|u\|_{s, p}} & =\|u\|_{s, p}^{p-1}-\frac{1}{\|u\|_{s, p}} \int_{\Omega} \tilde{f}(x, u) u d x \\
& \geqslant\|u\|_{s, p}^{p-1}-\frac{C}{\|u\|_{s, p}} \int_{\Omega}\left(1+|\underline{u}|^{q-1}+|\bar{u}|^{q-1}\right)|u| d x \\
& \geqslant\|u\|_{s, p}^{p-1}-\frac{C}{\|u\|_{s, p}}\left(\|u\|_{1}+\|\underline{u}\|_{q}^{q-1}\|u\|_{q}+\|\bar{u}\|_{q}^{q-1}\|u\|_{q}\right) \\
& \geqslant\|u\|_{s, p}^{p-1}-C,
\end{aligned}
$$

and the latter tends to $\infty$ as $\|u\|_{s, p} \rightarrow \infty$ (here we have used the continuous embeddings $W_{0}^{s, p}(\Omega) \hookrightarrow$ $\left.L^{1}(\Omega), L^{q}(\Omega)\right)$. By [8, Theorem 2.99], the equation

$$
A(u)+B(u)=0 \text { in } W^{-s, p^{\prime}}(\Omega)
$$

has a solution $u \in W_{0}^{s, p}(\Omega)$. Now we prove that in $\Omega$

$$
\underline{u} \leqslant u \leqslant \bar{u} .
$$

Clearly (3.7) holds in $\Omega^{c}$. Testing (3.6) with $(u-\bar{u})^{+} \in W_{0}^{s, p}(\Omega)_{+}$we have

$$
\begin{aligned}
\left\langle(-\Delta)_{p}^{s} u,(u-\bar{u})^{+}\right\rangle & =\int_{\Omega} \tilde{f}(x, u)(u-\bar{u})^{+} d x \\
& =\int_{\Omega} f(x, \bar{u})(u-\bar{u})^{+} d x \\
& \leqslant\left\langle(-\Delta)_{p}^{s} \bar{u},(u-\bar{u})^{+}\right\rangle
\end{aligned}
$$

where we also used that $\bar{u}$ is a supersolution of (1.1), so

$$
\left\langle(-\Delta)_{p}^{s} u-(-\Delta)_{p}^{s} \bar{u},(u-\bar{u})^{+}\right\rangle \leqslant 0 .
$$

By [4, Lemma A.2] and [26, Lemma 2.3] (with $g(t)=t^{+}$) we have for all $a, b \in \mathbb{R}$

$$
\left|a^{+}-b^{+}\right|^{p} \leqslant(a-b)^{p-1}\left(a^{+}-b^{+}\right), \quad(a-b)^{p-1} \leqslant C\left(a^{p-1}-b^{p-1}\right),
$$


hence

$$
\begin{aligned}
& \left\|(u-\bar{u})^{+}\right\|_{s, p}^{p}=\iint_{\mathbb{R}^{N} \times \mathbb{R}^{N}}\left|(u(x)-\bar{u}(x))^{+}-(u(y)-\bar{u}(y))^{+}\right|^{p} d \mu \\
& \leqslant \iint_{\mathbb{R}^{N} \times \mathbb{R}^{N}}[(u(x)-\bar{u}(x))-(u(y)-\bar{u}(y))]^{p-1}\left[(u(x)-\bar{u}(x))^{+}-(u(y)-\bar{u}(y))^{+}\right] d \mu \\
& \leqslant C \iint_{\mathbb{R}^{N} \times \mathbb{R}^{N}}\left[(u(x)-u(y))^{p-1}-(\bar{u}(x)-\bar{u}(y))^{p-1}\right]\left[(u(x)-\bar{u}(x))^{+}-(u(y)-\bar{u}(y))^{+}\right] d \mu \\
& =C\left\langle(-\Delta)_{p}^{s} u-(-\Delta)_{p}^{s} \bar{u},(u-\bar{u})^{+}\right\rangle \leqslant 0,
\end{aligned}
$$

so $(u-\bar{u})^{+}=0$, i.e., $u \leqslant \bar{u}$ in $\Omega$. Similarly we prove $u \geqslant \underline{u}$ and achieve (3.7). Finally, using (3.7) in (3.6) we see that $u \in W_{0}^{s, p}(\Omega)$ solves (1.1). Thus $u \in \mathcal{S}(\underline{u}, \bar{u})$.

We recall that a partially ordered set $(S, \leqslant)$ is downward directed (resp., upward directed) if for all $u_{1}, u_{2} \in S$ there exists $u_{3} \in S$ s.t. $u_{3} \leqslant u_{1}, u_{2}$ (resp., $u_{3} \geqslant u_{1}, u_{2}$ ), and that $S$ is directed if it is both downward and upward directed.

Lemma 3.3. Let $\mathbf{H}_{0}$ hold, $(\underline{u}, \bar{u})$ be a sub-supersolution pair of (1.1). Then, $\mathcal{S}(\underline{u}, \bar{u})$ is directed.

Proof. We prove that $\mathcal{S}(\underline{u}, \bar{u})$ is downward directed. Let $u_{1}, u_{2} \in \mathcal{S}(\underline{u}, \bar{u})$, then in particular $u_{1}, u_{2}$ are supersolutions of (1.1). Set $\hat{u}=u_{1} \wedge u_{2} \in W_{0}^{s, p}(\Omega)$, then by Lemma $3.1 \hat{u}$ is a supersolution of (1.1) and $\underline{u} \leqslant \hat{u}$. By Lemma 3.2 there exists $u_{3} \in \mathcal{S}(\underline{u}, \hat{u})$, in particular $u_{3} \in \mathcal{S}(\underline{u}, \bar{u})$ and $u_{3} \leqslant u_{1} \wedge u_{2}$.

Similarly we see that $\mathcal{S}(\underline{u}, \bar{u})$ is upward directed.

Another important property of $\mathcal{S}(\underline{u}, \bar{u})$ is compactness:

Lemma 3.4. Let $\mathbf{H}_{0}$ hold, $(\underline{u}, \bar{u})$ be a sub-supersolution pair of $(1.1)$. Then, $\mathcal{S}(\underline{u}, \bar{u})$ is compact in $W_{0}^{s, p}(\Omega)$. Proof. Let $\left(u_{n}\right)$ be a sequence in $\mathcal{S}(\underline{u}, \bar{u})$, then for all $n \in \mathbb{N}, v \in W_{0}^{s, p}(\Omega)$

$$
\left\langle(-\Delta)_{p}^{s} u_{n}, v\right\rangle=\int_{\Omega} f\left(x, u_{n}\right) v d x
$$

and $\underline{u} \leqslant u_{n} \leqslant \bar{u}$. Testing (3.8) with $u_{n} \in W_{0}^{s, p}(\Omega)$, we have by $\mathbf{H}_{0}$

$$
\begin{aligned}
\left\|u_{n}\right\|_{s, p}^{p} & =\int_{\Omega} f\left(x, u_{n}\right) u_{n} d x \\
& \leqslant c_{0} \int_{\Omega}\left(\left|u_{n}\right|+\left|u_{n}\right|^{q}\right) d x \\
& \leqslant c_{0}\left(\|\underline{u}\|_{1}+\|\bar{u}\|_{1}+\|\underline{u}\|_{q}^{q}+\|\bar{u}\|_{q}^{q}\right) \leqslant C,
\end{aligned}
$$

hence $\left(u_{n}\right)$ is bounded in $W_{0}^{s, p}(\Omega)$. Passing to a subsequence, we have $u_{n} \rightarrow u$ in $W_{0}^{s, p}(\Omega), u_{n}(x) \rightarrow u(x)$ and $\left|u_{n}(x)\right| \leqslant h(x)$ for a.e. $x \in \mathbb{N}$, with $h \in L^{q}(\Omega)$. Therefore,

$$
\begin{aligned}
\left|f\left(x, u_{n}\right)\left(u_{n}-u\right)\right| & \leqslant c_{0}\left(1+\left|u_{n}\right|^{q-1}\right)\left|u_{n}-u\right| \\
& \leqslant 2 c_{0}\left(1+g(x)^{q-1}\right)(|\underline{u}|+|\bar{u}|) \in L^{1}(\Omega) .
\end{aligned}
$$

Testing (3.8) with $u_{n}-u \in W_{0}^{s, p}(\Omega)$, we get

$$
\left\langle(-\Delta)_{p}^{s}\left(u_{n}\right), u_{n}-u\right\rangle=\int_{\Omega} f\left(x, u_{n}\right)\left(u_{n}-u\right) d x,
$$

and the latter tends to 0 as $n \rightarrow \infty$. By Lemma 2.1 we have $u_{n} \rightarrow u$ in $W_{0}^{s, p}(\Omega)$. Then, we can pass to the limit in (3.8) and conclude that $u \in \mathcal{S}(\underline{u}, \bar{u})$.

The main result of this section states that $\mathcal{S}(\underline{u}, \bar{u})$ contains extremal elements with respect to the pointwise ordering:

Theorem 3.5. Let $\mathbf{H}_{0}$ hold, $(\underline{u}, \bar{u})$ be a sub-supersolution pair of (1.1). Then $\mathcal{S}(\underline{u}, \bar{u})$ contains a smallest and a biggest element. 
Proof. The set $\mathcal{S}(\underline{u}, \bar{u})$ is bounded in both $W_{0}^{s, p}(\Omega)$ and $C_{s}^{\alpha}(\bar{\Omega})$. Indeed, for all $u \in \mathcal{S}(\underline{u}, \bar{u})$, testing (1.1) with $u \in W_{0}^{s, p}(\Omega)$ we have

$$
\begin{aligned}
\|u\|_{s, p}^{p} & =\int_{\Omega} f(x, u) u d x \\
& \leqslant c_{0} \int_{\Omega}\left(|u|+|u|^{q}\right) d x \\
& \leqslant c_{0}\left(\|\underline{u}\|_{1}+\|\bar{u}\|_{1}+\|\underline{u}\|_{q}^{q}+\|\bar{u}\|_{q}^{q}\right),
\end{aligned}
$$

hence $\mathcal{S}(\underline{u}, \bar{u})$ is bounded in $W_{0}^{s, p}(\Omega)$. Further, by Lemma 2.4, for all $u \in \mathcal{S}(\underline{u}, \bar{u})$ we have $u \in L^{\infty}(\Omega)$, $\|u\|_{\infty} \leqslant C$ (with $C=C(\underline{u}, \bar{u})>0$, here and in the forthcoming bounds). In turn, this implies $\|f(\cdot, u)\|_{\infty} \leqslant C$. Then we apply Lemma 2.5 (with $g=f(\cdot, u)$ ) to see that $u \in C_{s}^{\alpha}(\bar{\Omega}),\|u\|_{\alpha, s} \leqslant C$. So, $\mathcal{S}(\underline{u}, \bar{u})$ is bounded in $C_{s}^{\alpha}(\bar{\Omega})$ as well (in particular, then, $\mathcal{S}(\underline{u}, \bar{u})$ is equibounded in $\Omega$ ).

Now we prove that $\mathcal{S}(\underline{u}, \bar{u})$ has a minimum. Let $\left(x_{k}\right)$ be a dense subset of $\Omega$, and set

$$
m_{k}=\inf _{u \in \mathcal{S}(\underline{u}, \bar{u})} u\left(x_{k}\right)>-\infty
$$

for each $k \geqslant 1$ (recall $\mathcal{S}(\underline{u}, \bar{u})$ is equibounded). For all $n \in \mathbb{N}, k \in\{1, \ldots, n\}$ we can find $u_{n, k} \in \mathcal{S}(\underline{u}, \bar{u})$ s.t.

$$
u_{n, k}\left(x_{k}\right) \leqslant m_{k}+\frac{1}{n} \text {. }
$$

Since $\mathcal{S}(\underline{u}, \bar{u})$ is downward directed (Lemma 3.3), we can find $u_{n} \in \mathcal{S}(\underline{u}, \bar{u})$ s.t. $u_{n} \leqslant u_{n, k}$ for all $k \in\{1, \ldots, n\}$. In particular, for all $n \in \mathbb{N}, k \in\{1, \ldots, n\}$ we have

$$
u_{n}\left(x_{k}\right) \leqslant m_{k}+\frac{1}{n} .
$$

Since $\mathcal{S}(\underline{u}, \bar{u})$ is compact (Lemma 3.4), passing to a subsequence we have $u_{n} \rightarrow u_{0}$ in $W_{0}^{s, p}(\Omega)$ for some $u_{0} \in \mathcal{S}(\underline{u}, \bar{u})$. Besides, $\left(u_{n}\right) \subseteq \mathcal{S}(\underline{u}, \bar{u})$ is bounded in $C_{s}^{\alpha}(\bar{\Omega})$, hence up to a further subsequence $u_{n} \rightarrow u_{0}$ in $C_{s}^{0}(\bar{\Omega})$, in particular $u_{n}(x) \rightarrow u_{0}(x)$ for all $x \in \bar{\Omega}$. By (3.9) we have for all $k \in \mathbb{N}$

$$
u_{0}\left(x_{k}\right)=\lim _{n} u_{n}\left(x_{k}\right) \leqslant \lim _{n}\left(m_{k}+\frac{1}{n}\right)=m_{k} .
$$

Therefore, given $u \in \mathcal{S}(\underline{u}, \bar{u})$ we have $u_{0}\left(x_{k}\right) \leqslant u\left(x_{k}\right)$ for all $k \geqslant 1$, which by density of $\left(x_{k}\right)$ implies $u_{0} \leqslant u$. Hence,

$$
u_{0}=\min \mathcal{S}(\underline{u}, \bar{u}) .
$$

Similarly we prove the existence of $\max \mathcal{S}(\underline{u}, \bar{u})$.

Remark 3.6. For the sake of completeness, we recall that Theorem 3.5 can be proved following closely the proof of [8, Theorem 3.11], using Lemmas 3.3, 3.4, and the fact that $W_{0}^{s, p}(\Omega)$ is separable (another way consists in applying Zorn's Lemma, as in [8, Remark 3.12]). We also note the remark that, as seen in the proof of Theorem $3.5, \mathcal{S}(\underline{u}, \bar{u})$ turns out to be compact in $C_{s}^{0}(\bar{\Omega})$.

\section{Extremal constant Sign SOlutions}

In this section we prove that (1.1) has a smallest positive and a biggest negative solution (following the ideas of [9]), under the following hypotheses on $f$ :

$\mathbf{H}_{1} f: \Omega \times \mathbb{R} \rightarrow \mathbb{R}$ is a Carathéodory function, for all $(x, t) \in \Omega \times \mathbb{R}$ we set

$$
F(x, t)=\int_{0}^{t} f(x, \tau) d \tau,
$$

and the following conditions hold:

(i) $|f(x, t)| \leq c_{0}\left(1+|t|^{q-1}\right)$ for all a.e. $x \in \Omega$ and all $t \in \mathbb{R}\left(c_{0}>0, q \in\left(p, p_{s}^{*}\right)\right)$;

(ii) $\limsup _{|t| \rightarrow \infty} \frac{F(x, t)}{|t|^{p}}<\frac{\lambda_{1}}{p}$ uniformly for a.e. $x \in \Omega$;

(iii) $\lambda_{1}<\liminf _{t \rightarrow 0} \frac{f(x, t)}{|t|^{p-2} t} \leqslant \limsup _{t \rightarrow 0} \frac{f(x, t)}{|t|^{p-2} t}<\infty$ uniformly for a.e. $x \in \Omega$. 
Clearly $\mathbf{H}_{1}$ implies $\mathbf{H}_{0}$. Here $\lambda_{1}>0$ denotes the principal eigenvalue of $(-\Delta)_{p}^{s}$ in $W_{0}^{s, p}(\Omega)$, with associated positive, $L^{p}(\Omega)$-normalized eigenfunction $\hat{u}_{1} \in W_{0}^{s, p}(\Omega)$ (see Lemma $2.8(i)$ ). Note that by $\mathbf{H}_{1}$ (iii) we have $f(\cdot, 0)=0$ in $\Omega$, hence (1.1) has the trivial solution 0. Condition $\mathbf{H}_{1}($ iii $)$ conjures a $(p-1)$-linear behavior of $f(x, \cdot)$ near the origin.

In this and the forthcoming section, our approach to problem (1.1) is purely variational. Our result is the following:

Theorem 4.1. Let $\mathbf{H}_{1}$ hold. Then, (1.1) has a smallest positive solution $u_{+} \in \operatorname{int}\left(C_{s}^{0}(\bar{\Omega})_{+}\right)$and a biggest negative solution $u_{-} \in-\operatorname{int}\left(C_{s}^{0}(\bar{\Omega})_{+}\right)$.

Proof. We focus on positive solutions. Set for all $(x, t) \in \Omega \times \mathbb{R}$

$$
f_{+}(x, t)=f\left(x, t^{+}\right), \quad F_{+}(x, t)=\int_{0}^{t} f_{+}(x, \tau) d \tau,
$$

and for all $u \in W_{0}^{s, p}(\Omega)$

$$
\Phi_{+}(u)=\frac{\|u\|_{s, p}^{p}}{p}-\int_{\Omega} F_{+}(x, u) d x .
$$

Since $f_{+}(x, t)=0$ for all $(x, t) \in \Omega \times \mathbb{R}^{-}, f_{+}$satisfies $\mathbf{H}_{1}$ (with $t \rightarrow 0^{+}$in $($iii $)$). Therefore, $\Phi_{+} \in C^{1}\left(W_{0}^{s, p}(\Omega)\right.$ ). By $\mathbf{H}_{1}(i)$ and the compact embedding $W_{0}^{s, p}(\Omega) \hookrightarrow L^{q}(\Omega)$, it is easily seen that $\Phi_{+}$is sequentially weakly lower semicontinuous in $W_{0}^{s, p}(\Omega)$.

By $\mathbf{H}_{1}$ (ii) there exist $\theta \in\left(0, \lambda_{1}\right), K>0$ s.t. for a.e. $x \in \Omega$ and all $|t| \geqslant K$

$$
F_{+}(x, t) \leqslant \frac{\theta}{p}|t|^{p}
$$

Besides, by $\mathbf{H}_{\mathbf{1}}(i)$ we can find $C_{K}>0$ s.t. for a.e. $x \in \Omega$ and all $t \in \mathbb{R}$

$$
F_{+}(x, t) \leqslant \frac{\theta}{p}|t|^{p}+C_{K}
$$

So, for all $u \in W_{0}^{s, p}(\Omega)$ we have

$$
\begin{aligned}
\Phi_{+}(u) & \geqslant \frac{\|u\|_{s, p}^{p}}{p}-\int_{\Omega}\left(\frac{\theta}{p}|u|^{p}+C_{K}\right) d x \\
& \geqslant \frac{\|u\|_{s, p}^{p}}{p}-\frac{\theta}{p}\|u\|_{p}^{p}-C_{K}|\Omega| \\
& \geqslant\left(1-\frac{\theta}{\lambda_{1}}\right) \frac{\|u\|_{s, p}^{p}}{p}-C_{K}|\Omega|
\end{aligned}
$$

(where we used Lemma 2.8), and the latter tends to infinity as $\|u\|_{s, p} \rightarrow \infty$. Therefore $\Phi_{+}$is coercive. Thus, there is $\hat{u} \in W_{0}^{s, p}(\Omega)$ s.t.

$$
\Phi_{+}(\hat{u})=\inf _{u \in W_{0}^{s, p}(\Omega)} \Phi_{+}(u) .
$$

In particular, we have $\Phi_{+}^{\prime}(\hat{u})=0$, i.e.,

$$
(-\Delta)_{p}^{s} \hat{u}=f_{+}(\cdot, \hat{u}) \text { in } W^{-s, p^{\prime}}(\Omega) .
$$

Testing (4.2) with $-\hat{u}^{-} \in W_{0}^{s, p}(\Omega)$, we get

$$
\left\|\hat{u}^{-}\right\|^{p} \leqslant-\left\langle(-\Delta)_{p}^{s} \hat{u}, \hat{u}^{-}\right\rangle=-\int_{\Omega} f_{+}(x, \hat{u}) \hat{u}^{-} d x=0,
$$

so $\hat{u} \geqslant 0$. Hence, $f_{+}(\cdot, \hat{u})=f(\cdot, \hat{u})$, therefore $(4.2)$ rephrases as

$$
(-\Delta)_{p}^{s}(\hat{u})=f(\cdot, \hat{u}) \text { in } W^{-s, p^{\prime}}(\Omega),
$$

i.e., $\hat{u} \in W_{0}^{s, p}(\Omega)_{+}$is a solution of (1.1). By Lemmas 2.4, 2.5 we have $\hat{u} \in C_{s}^{0}(\bar{\Omega})_{+}$. By $\mathbf{H}_{1}($ iii), we can find $\lambda_{1}<c_{1}<c_{2}, \delta>0$ s.t. for a.e. $x \in \Omega$ and all $t \in[0, \delta]$

$$
c_{1} t^{p-1} \leqslant f(x, t) \leqslant c_{2} t^{p-1} .
$$


Choose $\tau>0$ s.t. $0<\tau \hat{u}_{1} \leqslant \delta$ in $\Omega$. Then by (4.1), (4.3), and Lemma 2.8 we have

$$
\begin{aligned}
\Phi_{+}(\hat{u}) & \leqslant \Phi_{+}\left(\tau \hat{u}_{1}\right) \\
& =\frac{\tau^{p}}{p}\left\|\hat{u}_{1}\right\|_{s, p}^{p}-\int_{\Omega} F_{+}\left(x, \tau \hat{u}_{1}\right) d x \\
& \leqslant \frac{\tau^{p}}{p}\left\|\hat{u}_{1}\right\|_{s, p}^{p}-\frac{\tau^{p} c_{1}}{p}\left\|\hat{u}_{1}\right\|_{p}^{p} \\
& =\frac{\tau^{p}}{p}\left(\lambda_{1}-c_{1}\right)<0,
\end{aligned}
$$

hence $\hat{u} \neq 0$. By (4.2), (4.3) we have for all $v \in W_{0}^{s, p}(\Omega)_{+}$

$$
\begin{aligned}
\left\langle(-\Delta)_{p}^{s} \hat{u}, v\right\rangle & \geqslant \int_{\{\hat{u} \leqslant \delta\}} c_{1} \hat{u}^{p-1} v d x-\int_{\{\hat{u}>\delta\}} c_{0}\left(1+\hat{u}^{q-1}\right) v d x \\
& \geqslant \int_{\Omega} c_{1} \hat{u}^{p-1} v d x-c_{0} \int_{\{\hat{u}>\delta\}}\left[\frac{1}{\delta^{p-1}}+\|\hat{u}\|_{\infty}^{q-p}\right] \hat{u}^{p-1} v d x \\
& \geqslant-C \int_{\Omega} \hat{u}^{p-1} v d x
\end{aligned}
$$

for some $C>0$. By Lemma 2.7 and (2.1) we have $\hat{u} \in \operatorname{int}\left(C_{s}^{0}(\bar{\Omega})_{+}\right)$, so there is $r>0$ s.t. $u \in C_{s}^{0}(\bar{\Omega})_{+}$for all $u \in C_{s}^{0}(\bar{\Omega})$ with $\|u-\hat{u}\|_{0, s}<r$. Now pick

$$
0<\varepsilon<\min \left\{\frac{\delta}{\|\hat{u}\|_{\infty}}, \frac{r}{\left\|\hat{u}_{1}\right\|_{0, s}}\right\} .
$$

By (4.3) we have for all $v \in W_{0}^{s, p}(\Omega)_{+}$

$$
\left\langle(-\Delta)_{p}^{s}\left(\varepsilon \hat{u}_{1}\right), v\right\rangle=\lambda_{1} \int_{\Omega}\left(\varepsilon \hat{u}_{1}\right)^{p-1} v d x \leqslant \int_{\Omega} f\left(x, \varepsilon \hat{u}_{1}\right) v d x,
$$

hence $\varepsilon \hat{u}_{1}$ is a subsolution of (1.1). Besides,

$$
\left\|\left(\hat{u}-\varepsilon \hat{u}_{1}\right)-\hat{u}\right\|_{0, s}=\varepsilon\left\|\hat{u}_{1}\right\|_{0, s}<r,
$$

so $\hat{u}-\varepsilon \hat{u}_{1} \in C_{s}^{0}(\bar{\Omega})_{+}$, in particular $\varepsilon \hat{u}_{1} \leqslant \hat{u}$. Therefore $\left(\varepsilon \hat{u}_{1}, \hat{u}\right)$ is a sub-supersolution pair of (1.1).

For all $n \in \mathbb{N}$ big enough, $\varepsilon=\frac{1}{n}$ satisfies (4.4). By Theorem 3.5, there exists

$$
u_{n}=\min \mathcal{S}\left(\frac{\hat{u}_{1}}{n}, \hat{u}\right) \text {. }
$$

Clearly $(0, \hat{u})$ is a sub-supersolution pair of $(1.1)$ and $u_{n} \in \mathcal{S}(0, \hat{u})$, so by Lemma 3.4, passing if necessary to a subsequence, we have $u_{n} \rightarrow u_{+}$in $W_{0}^{s, p}(\Omega)$ for some $u_{+} \in \mathcal{S}(0, \hat{u})$.

On the other hand we have for all $n \in \mathbb{N}$

$$
\mathcal{S}\left(\frac{\hat{u}_{1}}{n}, \hat{u}\right) \subseteq \mathcal{S}\left(\frac{\hat{u}_{1}}{n+1}, \hat{u}\right),
$$

hence by minimality $u_{n+1} \leqslant u_{n}$. This in turn implies that $u_{n}(x) \rightarrow u_{+}(x)$ for a.e. $x \in \Omega$. Now, since $0 \leqslant u_{n} \leqslant \hat{u}$, we see that $\left(u_{n}\right)$ is a bounded sequence in $L^{\infty}(\Omega)$, hence by $\mathbf{H}_{1}(i)\left(f\left(\cdot, u_{n}\right)\right)$ is uniformly bounded as well. Then, since for all $n \in \mathbb{N}$

$$
(-\Delta)_{p}^{s} u_{n}=f\left(\cdot, u_{n}\right) \text { in } W^{-s, p^{\prime}}(\Omega),
$$

Lemmas 2.4, 2.5 imply that $\left(u_{n}\right)$ is bounded in $C_{s}^{\alpha}(\bar{\Omega})$ as well. So, passing to a further subsequence, we have $u_{n} \rightarrow u_{+}$in $C_{s}^{0}(\bar{\Omega})$.

We prove now that $u_{+} \neq 0$, by contradiction. If $u_{+}=0$, then $u_{n} \rightarrow 0$ uniformly in $\bar{\Omega}$. Set

$$
v_{n}=\frac{u_{n}}{\left\|u_{n}\right\|_{s, p}} \in W_{0}^{s, p}(\Omega)_{+},
$$

then by (4.5) we have for all $n \in \mathbb{N}$

$$
(-\Delta)_{p}^{s} v_{n}=\frac{f\left(\cdot, u_{n}\right)}{\left\|u_{n}\right\|_{s, p}^{p-1}}=\frac{f\left(\cdot, u_{n}\right)}{u_{n}^{p-1}} v_{n}^{p-1} \text { in } W^{-s, p^{\prime}}(\Omega) .
$$


Set for all $n \in \mathbb{N}$

$$
\rho_{n}=\frac{f\left(\cdot, u_{n}\right)}{u_{n}^{p-1}}
$$

By (4.3), for $n \in \mathbb{N}$ big enough we have $c_{1} \leqslant \rho_{n} \leqslant c_{2}$ in $\Omega$, in particular $\rho_{n} \in L^{\infty}(\Omega)$. Then $v_{n} \in W_{0}^{s, p}(\Omega) \backslash\{0\}$ is an eigenfunction of the (2.4)-type eigenvalue problem

$$
(-\Delta)_{p}^{s} v_{n}=\lambda \rho_{n} v_{n}^{p-1} \text { in } W^{-s, p^{\prime}}(\Omega),
$$

associated with the eigenvalue $\lambda=1$. Since $\rho_{n} \geqslant c_{1}>\lambda_{1}$, by Lemma 2.8 (iii) we have

$$
\lambda_{1}\left(\rho_{n}\right)<\lambda_{1}\left(\lambda_{1}\right)=1,
$$

therefore $v_{n}$ is a non-principal eigenfunction of (4.6). By Lemma 2.8 (ii) $v_{n}$ is nodal, a contradiction. Hence, by Lemma 2.7 and $(2.1)$ we have $u_{+} \in \operatorname{int}\left(C_{s}^{0}(\bar{\Omega})_{+}\right)$.

Finally, we prove that $u_{+}$is the smallest positive solution of (1.1). Let $u \in W_{0}^{s, p}(\Omega)_{+} \backslash\{0\}$ be a solution of (1.1). Arguing as above we see that $u \in \operatorname{int}\left(C_{s}^{0}(\bar{\Omega})_{+}\right)$. Set $w=u \wedge \hat{u} \in W_{0}^{s, p}(\Omega)_{+}$, then by Lemma $3.1 w$ is a supersolution of (1.1). As above, for all $n \in \mathbb{N}$ big enough we have that $\frac{\hat{u}_{1}}{n}$ is a subsolution of (1.1) and $\frac{\hat{u}_{1}}{n} \leqslant w$ in $\Omega$, i.e., $\left(\hat{u}_{1} / n, w\right)$ is a sub-supersolution pair. Therefore, by Lemma 3.2 we can find

Since

$$
w_{n} \in \mathcal{S}\left(\frac{\hat{u}_{1}}{n}, w\right) \text {. }
$$

$$
\mathcal{S}\left(\frac{\hat{u}_{1}}{n}, w\right) \subseteq \mathcal{S}\left(\frac{\hat{u}_{1}}{n}, \hat{u}\right),
$$

by minimality, for all $n \in \mathbb{N}$ big enough we have $u_{n} \leqslant w_{n}$, hence $u_{n} \leqslant u$. Passing to the limit as $n \rightarrow \infty$, we have $u_{+} \leqslant u$.

Similarly we prove existence of the biggest negative solution $u_{-} \in-\operatorname{int}\left(C_{s}^{0}(\bar{\Omega})_{+}\right)$.

Remark 4.2. According to [21], most properties on Lemma 2.8 also hold if $\rho$ lies in a special class $\widetilde{W}_{p}$ of singular weights, namely if $\rho \mathrm{d}_{\Omega}^{s a} \in L^{r}(\Omega)$ for some $a \in[0,1], r>1$ satisfying

$$
\frac{1}{r}+\frac{a}{p}+\frac{p-a}{p_{s}^{*}}<1
$$

So, in view of the proof of Theorem 4.1 above, a natural question is whether we may replace $\mathbf{H}_{1}$ (iii) with the weaker condition

$$
\liminf _{t \rightarrow 0} \frac{f(x, t)}{t^{p-1}}>\lambda_{1} \text { uniformly for a.e. } x \in \Omega \text {. }
$$

Define $\rho_{n}=f\left(\cdot, u_{n}\right) / u_{n}^{p-1}$ as above, then recalling that $u_{n} \geqslant c \mathrm{~d}_{\Omega}^{s}$ in $\bar{\Omega}$ we have

$$
0<\rho_{n} \leqslant C\left(1+d_{\Omega}^{-s(p+1)}\right) .
$$

Unfortunately, this does not ensure that $\rho_{n} \in \widetilde{W}_{p}$, in general. For instance, consider the case $\Omega=B_{1}(0)$, $\mathrm{d}_{\Omega}(x)=1-|x|$. Then we have $\mathrm{d}_{\Omega}^{s} \in L^{\alpha}(\Omega)$ iff $\alpha \in(0,1)$. Therefore, $\rho_{n} \in \widetilde{W}_{p}$ implies

$$
\left\{\begin{array}{l}
s r(p-a-1)<1 \\
\frac{1}{r}+\frac{a}{p}+\frac{p-a}{p_{s}^{*}}<1,
\end{array}\right.
$$

in particular $(p-2) s<1$. Yet, for special values of $p, s$, and a suitable domain $\Omega$, analogues to Theorem 4.1 could be proved for reactions $f(x, \cdot)$ with a $(p-1)$-sublinear behavior near the origin.

\section{NODAL SOLUTiOnS}

In this section we present an application of our main result, following the ideas of [16] (see also [34, Theorem 11.26]). Applying Theorem 4.1, along with the mountain pass theorem and spectral theory for $(-\Delta)_{p}^{s}$, we prove existence of a nodal solution of (1.1). Our hypotheses on the reaction $f$ are the following:

$\mathbf{H}_{2} f: \Omega \times \mathbb{R} \rightarrow \mathbb{R}$ is a Carathéodory function, for all $(x, t) \in \Omega \times \mathbb{R}$ we set

$$
F(x, t)=\int_{0}^{t} f(x, \tau) d \tau
$$

and the following conditions hold: 
(i) $|f(x, t)| \leq c_{0}\left(1+|t|^{q-1}\right)$ for all a.e. $x \in \Omega$ and all $t \in \mathbb{R}\left(c_{0}>0, q \in\left(p, p_{s}^{*}\right)\right)$;

(ii) $\limsup _{|t| \rightarrow \infty} \frac{F(x, t)}{|t|^{p}}<\frac{\lambda_{1}}{p}$ uniformly for a.e. $x \in \Omega$;

(iii) $\lambda_{2}<\liminf _{t \rightarrow 0} \frac{f(x, t)}{|t|^{p-2} t} \leqslant \limsup _{t \rightarrow 0} \frac{f(x, t)}{|t|^{p-2} t}<\infty$ uniformly for a.e. $x \in \Omega$.

Here $\lambda_{2}>\lambda_{1}$ denotes the second (variational) eigenvalue of $(-\Delta)_{p}^{s}$ in $W_{0}^{s, p}(\Omega)$, defined by (2.5). Again, we are assuming for $f(x, \cdot)$ a $(p-1)$-linear behavior near the origin.

Our method is variational. We define the energy functional $\Phi$ as in Section 1 and recall the following Palais-Smale compactness condition:

(PS) Any sequence $\left(u_{n}\right)_{n}$ in $W_{0}^{s, p}(\Omega)$, s.t. $\left(\Phi\left(u_{n}\right)\right)$ is bounded in $\mathbb{R}$ and $\Phi^{\prime}\left(u_{n}\right) \rightarrow 0$ in $W^{-s, p^{\prime}}$, admits a (strongly) convergent subsequence.

We will use the following notation for critical points:

$$
K(\Phi)=\left\{u \in W_{0}^{s, p}(\Omega): \Phi^{\prime}(u)=0 \text { in } W^{-s, p^{\prime}}(\Omega)\right\}
$$

and for all $c \in \mathbb{R}$

$$
K_{c}(\Phi)=\{u \in K(\Phi): \Phi(u)=c\}
$$

Our result is the following:

Theorem 5.1. Let $\mathbf{H}_{2}$ hold. Then, (1.1) has a smallest positive solution $u_{+} \in \operatorname{int}\left(C_{s}^{0}(\bar{\Omega})_{+}\right)$, a biggest negative solution $u_{-} \in-\operatorname{int}\left(C_{s}^{0}(\bar{\Omega})_{+}\right)$, and a nodal solution $\tilde{u} \in C_{s}^{0}(\bar{\Omega})$ s.t. $u_{-} \leqslant \tilde{u} \leqslant u_{+}$in $\Omega$.

Proof. Clearly $\mathbf{H}_{2}$ implies $\mathbf{H}_{1}$. From Theorem 4.1, then, we know that (1.1) has a smallest positive solution $u_{+} \in \operatorname{int}\left(C_{s}^{0}(\bar{\Omega})_{+}\right)$and a biggest negative solution $u_{-} \in-\operatorname{int}\left(C_{s}^{0}(\bar{\Omega})_{+}\right)$. Plus, by $\mathbf{H}_{2}($ iii $), 0$ is a solution of (1.1). We are going to detect a fourth solution $\tilde{u} \in W_{0}^{s, p}(\Omega)$, and then show that it is nodal.

Set for all $(x, t) \in \Omega \times \mathbb{R}$

$$
\tilde{f}(x, t)= \begin{cases}f\left(x, u_{-}(x)\right) & \text { if } t<u_{-}(x) \\ f(x, t) & \text { if } u_{-}(x) \leqslant t \leqslant u_{+}(x) \\ f\left(x, u_{+}(x)\right) & \text { if } t>u_{+}\end{cases}
$$

and

$$
\tilde{F}(x, t)=\int_{0}^{t} \tilde{f}(x, \tau) d \tau .
$$

Since $u_{ \pm} \in L^{\infty}(\Omega), \tilde{f}$ satisfies $\mathbf{H}_{0}$. Now set for all $u \in W_{0}^{s, p}(\Omega)$

$$
\tilde{\Phi}(u)=\frac{\|u\|_{s, p}^{p}}{p}-\int_{\Omega} \tilde{F}(x, u) d x .
$$

By $\mathbf{H}_{2}(i)$ (ii), reasoning as in the proof of Theorem 4.1 we see that $\tilde{\Phi} \in C^{1}\left(W_{0}^{s, p}(\Omega)\right)$ is coercive. As a consequence, $\tilde{\Phi}$ satisfies (PS) (see [22, Proposition 2.1]). Whenever $u \in W_{0}^{s, p}(\Omega)$ is a critical point of $\tilde{\Phi}$, then for all $v \in W_{0}^{s, p}(\Omega)$

$$
\left\langle(-\Delta)_{p}^{s} u, v\right\rangle=\int_{\Omega} \tilde{f}(x, u) v d x .
$$

By Lemmas 2.4, 2.5 we have $u \in C_{s}^{0}(\bar{\Omega})$. Besides, testing (5.1) with $\left(u-u_{+}\right)^{+},-\left(u-u_{-}\right)^{-} \in W_{0}^{s, p}(\Omega)$ and arguing as in Lemma 3.2 we have $u_{-} \leqslant u \leqslant u_{+}$in $\Omega$, hence $u$ solves (1.1) in $\Omega$. Using the notation of Section 3 , we can say that $u \in \mathcal{S}\left(u_{-}, u_{+}\right)$.

We introduce a further truncation setting for all $(x, t) \in \Omega \times \mathbb{R}$

$$
\tilde{f}_{+}(x, t)=\tilde{f}\left(x, t^{+}\right), \quad \tilde{F}_{+}(x, t)=\int_{0}^{t} \tilde{f}_{+}(x, \tau) d \tau,
$$

and for all $u \in W_{0}^{s, p}(\Omega)$

$$
\tilde{\Phi}_{+}(u)=\frac{\|u\|_{s, p}^{p}}{p}-\int_{\Omega} \tilde{F}_{+}(x, u) d x .
$$


Reasoning as above, we see that $\tilde{\Phi}_{+} \in C^{1}\left(W_{0}^{s, p}(\Omega)\right)$ is coercive, and whenever $u \in W_{0}^{s, p}(\Omega)$ is a critical point of $\tilde{\Phi}_{+}$we have $u \in \mathcal{S}\left(0, u_{+}\right)$. By the compact embedding $W_{0}^{s, p}(\Omega) \hookrightarrow L^{q}(\Omega)$, it is easily seen that $\tilde{\Phi}_{+}$is sequentially weakly lower semicontinuous, hence there exists $\tilde{u}_{+} \in W_{0}^{s, p}(\Omega)$ s.t.

$$
\tilde{\Phi}_{+}\left(\tilde{u}_{+}\right)=\inf _{u \in W_{0}^{s, p}(\Omega)} \tilde{\Phi}_{+}(u) .
$$

Arguing as in Theorem 4.1 we see that $\tilde{\Phi}_{+}\left(\tilde{u}_{+}\right)<0$, hence $\tilde{u}_{+} \neq 0$. By $\mathbf{H}_{2}$ (iii) and Lemma 2.7, we have $\tilde{u}_{+} \in \operatorname{int}\left(C_{s}^{0}(\bar{\Omega})_{+}\right)$. So, $\tilde{u}_{+}$is a positive solution of $(1.1)$, hence the minimality of $u_{+}$implies $\tilde{u}_{+}=u_{+}$. In particular, since $\tilde{\Phi}=\tilde{\Phi}_{+}$in $C_{s}^{0}(\bar{\Omega})_{+}$, we see that $u_{+} \in \operatorname{int}\left(C_{s}^{0}(\bar{\Omega})_{+}\right)$is a local minimizer of $\tilde{\Phi}$ in $C_{s}^{0}(\bar{\Omega})$. By Lemma 2.6, then $u_{+}$is a local minimizer of $\tilde{\Phi}$ in $W_{0}^{s, p}(\Omega)$ as well (recall that $\tilde{f}$ sarisfies $\mathbf{H}_{0}$ ).

Similarly we prove that $u_{-} \in-\operatorname{int}\left(C_{s}^{0}(\bar{\Omega})_{+}\right)$is a local minimizer of $\tilde{\Phi}$.

Now we argue by contradiction, assuming that there are no other critical points of $\tilde{\Phi}$ than $0, u_{+}$, and $u_{-}$, namely,

$$
K(\tilde{\Phi})=\left\{0, u_{+}, u_{-}\right\} .
$$

In particular, both $u_{ \pm}$are strict local minimizers of $\tilde{\Phi}$, which satisfies (PS). By the mountain pass Theorem [34, Proposition 5.42], there exists $\tilde{u} \in K_{c}(\tilde{\Phi})$, where we have set

$$
\Gamma=\left\{\gamma \in C\left([0,1], W_{0}^{s, p}(\Omega)\right): \gamma(0)=u_{+}, \gamma(1)=u_{-}\right\},
$$

and

$$
c=\inf _{\gamma \in \Gamma} \max _{t \in[0,1]} \tilde{\Phi}(\gamma(t))>\max \left\{\tilde{\Phi}\left(u_{+}\right), \tilde{\Phi}\left(u_{-}\right)\right\} .
$$

In particular $\tilde{u} \neq u_{ \pm}$, which by (5.2) implies $\tilde{u}=0$ and hence $c=0$. Set

$$
\Sigma=\left\{u \in W_{0}^{s, p}(\Omega) \cup C_{s}^{0}(\bar{\Omega}):\|u\|_{p}=1\right\} .
$$

By $\mathbf{H}_{2}$ (iii) we can find $\mu>\lambda_{2}, \delta>0$ s.t. for all $x \in \Omega,|t| \leqslant \delta$

$$
F(x, t) \geqslant \frac{\mu}{p}|t|^{p} .
$$

By (2.5) there is $\gamma_{1} \in \Gamma_{1}$ s.t.

$$
\max _{t \in[0,1]}\left\|\gamma_{1}(t)\right\|_{s, p}^{p}<\mu
$$

and by density we may assume $\gamma_{1} \in C([0,1], \Sigma)$, continuous with respect to the $C_{s}^{0}(\bar{\Omega})$-norm (see [15] for details). Since $t \mapsto\left\|\gamma_{1}(t)\right\|_{\infty}$ is bounded in [0,1], we can find $\varepsilon>0$ s.t. $\left\|\varepsilon \gamma_{1}(t)\right\|_{\infty} \leqslant \delta$ for all $t \in[0,1]$.

Besides, taking $\varepsilon>0$ even smaller if necessary, we have for all $t \in[0,1]$

$$
u_{+}-\varepsilon_{t} \gamma_{1}(t) \in \operatorname{int}\left(C_{s}^{0}(\bar{\Omega})_{+}\right), \quad u_{-}-\varepsilon_{t} \gamma_{1}(t) \in-\operatorname{int}\left(C_{s}^{0}(\bar{\Omega})_{+}\right),
$$

in particular $u_{-}<\varepsilon \gamma_{1}(t)<u_{+}$a.e. in $\Omega$. So, for all $t \in[0,1]$ we get

$$
\begin{aligned}
\tilde{\Phi}\left(\varepsilon \gamma_{1}(t)\right) & =\frac{\varepsilon^{p}}{p}\left\|\gamma_{1}(t)\right\|_{s, p}^{p}-\int_{\Omega} \tilde{F}\left(x, \varepsilon \gamma_{1}(t)\right) d x \\
& \leqslant \frac{\varepsilon^{p}}{p}\left\|\gamma_{1}(t)\right\|_{s, p}^{p}-\frac{\mu \varepsilon^{p}}{p}\left\|\gamma_{1}(t)\right\|_{p}^{p} \\
& =\frac{\varepsilon^{p}}{p}\left(\left\|\gamma_{1}(t)\right\|_{s, p}^{p}-\mu\right)<0 .
\end{aligned}
$$

Thus, $\varepsilon \gamma_{1}$ is a continuous path joining $\varepsilon \hat{u}_{1}$ to $-\varepsilon \hat{u}_{1}$, s.t. for all $t \in[0,1]$

$$
\tilde{\Phi}\left(\varepsilon \gamma_{1}(t)\right)<0 \text {. }
$$

Besides, by (5.2) and Lemma 2.7 we have

$$
K\left(\tilde{\Phi}_{+}\right)=\left\{0, u_{+}\right\}
$$

Set $a=\tilde{\Phi}_{+}\left(u_{+}\right), b=\tilde{\Phi}_{+}\left(\varepsilon \hat{u}_{1}\right)$, hence $a<b<0$ and there is no critical level in $(a, b]$. Therefore, by the second deformation theorem [34, Theorem 5.34] there exists a continuous deformation $h:[0,1] \times\left\{\tilde{\Phi}_{+} \leqslant b\right\} \rightarrow$ $\left\{\tilde{\Phi}_{+} \leqslant b\right\}$ s.t. for all $t \in[0,1], \tilde{\Phi}_{+}(u) \leqslant b$

$$
h(0, u)=u, \quad h(1, u)=u_{+}, \quad \tilde{\Phi}_{+}(h(t, u)) \leqslant \tilde{\Phi}_{+}(u) .
$$


Set for all $t \in[0,1]$

$$
\gamma_{+}(t)=h\left(t, \varepsilon \hat{u}_{1}\right)^{+} \in W_{0}^{s, p}(\Omega)_{+},
$$

then $\gamma_{+} \in C\left([0,1], W_{0}^{s, p}(\Omega)\right)$ with $\gamma_{+}(0)=\varepsilon \hat{u}_{1}, \gamma(1)=u_{+}$, and for all $t \in[0,1]$

$$
\tilde{\Phi}\left(\gamma_{+}(t)\right) \leqslant b<0 \text {. }
$$

Similarly we construct $\gamma_{-} \in C\left([0,1], W_{0}^{s, p}(\Omega)\right)$ s.t. $\gamma_{-}(0)=-\varepsilon \hat{u}_{1}, \gamma(1)=u_{-}$, and for all $t \in[0,1]$

$$
\tilde{\Phi}\left(\gamma_{-}(t)\right)<0 \text {. }
$$

Concatenating $\gamma_{+}, \varepsilon \gamma_{1}, \gamma_{-}$we find a path $\gamma \in \Gamma$ s.t. for all $t \in[0,1]$

$$
\tilde{\Phi}(\gamma(t))<0,
$$

hence $c<0$, a contradiction. So, (5.2) is false, i.e., there exists $\tilde{u} \in K(\tilde{\Phi}) \backslash\left\{0, u_{+}, u_{-}\right\}$, so as ween above we have $\tilde{u} \in \mathcal{S}\left(u_{-}, u_{+}\right)$.

Finally, we prove that $\tilde{u}$ is nodal. Indeed, if $\tilde{u} \in W_{0}^{s, p}(\Omega)_{+} \backslash\{0\}$, then by Lemma 2.7 we would have $\tilde{u} \in \operatorname{int}\left(C_{s}^{0}(\bar{\Omega})_{+}\right)$, along with $\tilde{u} \leqslant u_{+}$, which, by Theorem 4.1, would imply $\tilde{u}=u_{+}$, a contradiction. Similarly we see that $\tilde{u}$ cannot be negative.

Thus, $\tilde{u} \in C_{s}^{0}(\bar{\Omega}) \backslash\{0\}$ is a nodal solution of (1.1) s.t. $u_{-} \leqslant \tilde{u} \leqslant u_{+}$a.e. in $\Omega$.

Remark 5.2. The argument based on the characterization of $\lambda_{2}$ was already employed in [26, Theorem 4.1] and [15, Theorem 3.3] (for $p=2$ ). The novelty of Theorem 5.1 above, with respect to such results (even for the linear case $p=2$ ), lies in the detailed information about solutions, as we prove that $u_{ \pm}$are extremal constant sign solutions and $\tilde{u}$ is nodal. We also remark that the assumption $p \geqslant 2$ is essentially due to regularity theory (Lemma 2.5), but the arguments displayed in this paper also work, with minor adjustments, for $p \in(1,2)$.

Acknowledgement. Both authors are members of GNAMPA (Gruppo Nazionale per l'Analisi Matematica, la Probabilità e le loro Applicazioni) of INdAM (Istituto Nazionale di Alta Matematica 'Francesco Severi'). A. Iannizzotto is supported by the grant PRIN n. 2017AYM8XW: Non-linear Differential Problems via Variational, Topological and Set-valued Methods, and by the research project Integro-differential Equations and nonlocal Problems funded by Fondazione di Sardegna (2017). We thank S. Mosconi for useful discussions.

\section{REFERENCES}

[1] A. Ambrosetti, D. Lupo, On a class of nonlinear Dirichlet problems with multiple solutions, Nonlinear Anal. 8 (1984) $1145-1150$.

[2] T. BARtsch, Z. Liu, On a superlinear elliptic $p$-Laplacian equation, J. Differential Equations 198 (2004) $149-175$.

[3] L. Brasco, E. Lindgren, Higher Sobolev regularity for the fractional $p$-Laplace equation in the superquadratic case. Adv. Math. 304 (2017) 300-354.

[4] L. Brasco, E. PArini, The second eigenvalue of the fractional p-Laplacian, Adv. Calc. Var. 9 (2016) 323-355.

[5] H. Brezis, L. Nirenberg, $H^{1}$ versus $C^{1}$ local minimizers, C. R. Acad. Sci. Paris Sér. I 317 (1993) 465-472.

[6] X. Cabré, Y. SiRe, Nonlinear equations for fractional Laplacians I: Regularity, maximum principles, and Hamiltonian estimates, Ann. Inst. Henri Poincaré (C) Nonlinear Analysis 31 (2014) 23-53.

[7] X. Cabré, Y. Sire, Nonlinear equations for fractional Laplacians II: Existence, uniqueness, and qualitative properties of solutions, Trans. Amer. Math. Soc. 367 (2015) 911-941.

[8] S. Carl, V. K. Le, D. Motreanu, Nonsmooth variational problems and their inequalities, Springer, New York (2007).

[9] S. Carl, K. Perera, Sign-changing and multiple solutions for the p-Laplacian, Abstr. Appl. Anal. 7 (2002) $613-625$.

[10] W. Chen, S. Mosconi, M. Squassina, Nonlocal problems with critical Hardy nonlinearity, J. Funct. Anal. 275 (2018) 3065-3114.

[11] E.N. Dancer, Y. Du, On sign-changing solutions of certain semilinear elliptic problems, Appl. Anal. 56 (1995) $193-206$.

[12] L.M. Del Pezzo, A. QuaAs, A Hopf's lemma and a strong minimum principle for the fractional $p$-Laplacian, J. Differential Eq. 263 (2017) 765-778.

[13] L.M. Del Pezzo, A. QuaAs, Global bifurcation for fractional p-Laplacian and an application, Z. Anal. Anwend. 35 (2016) 411-447.

[14] E. Di Nezza, G. Palatucci, E. Valdinoci, Hitchhiker's guide to the fractional Sobolev spaces, Bull. Sci. Math. 136 (2012) $521-573$.

[15] F.G. Düzgün, A. Iannizzotto, Three nontrivial solutions for nonlinear fractional Laplacian equations, Adv. Nonlinear Anal. 7 (2018) 211-226.

[16] M.E. Filippakis, N.S. Papageorgiou, Multiple constant sign and nodal solutions for nonlinear elliptic equations with the p-Laplacian, J. Differential Equations 245 (2008) 1883-1922.

[17] G. Franzina, G. Palatucci, Fractional p-eigenvalues, Riv. Mat. Univ. Parma 5 (2014) 373-386. 
[18] S. Frassu, E. M. Rocha, S. Santos, The obstacle problem at 0 for the fractional $p$-Laplacian, preprint.

[19] Y. Fu, P. PuCCI, Multiplicity existence for sublinear fractional Laplacian problems, Appl. Anal. 96 (2017) $1497-1508$.

[20] L. Gasiński, N.S. Papageorgiou, Extremal, nodal and stable solutions for nonlinear elliptic equations, Adv. Nonlinear Stud. 15 (2015) 629-665.

[21] K. Ho, I. Sim, Properties of eigenvalues and some regularities on fractional $p$-Laplacian with singular weights, preprint arXiv: 1809.07020.

[22] A. Iannizzotto, S. Liu, K. Perera, M. Squassina, Existence results for fractional $p$-Laplacian problems via Morse theory, Adv. Calc. Var. 9 (2016) 101-125.

[23] A. Iannizzotto, S. Mosconi, M. Squassina, $H^{s}$ versus $C^{0}$-weighted minimizers, Nonlinear Differ. Equ. Appl. 22 (2015) $477-497$

[24] A. Innnizzotto, S. Mosconi, M. Squassina, Global Hölder regularity for the fractional p-Laplacian, Rev. Mat. Iberoam. 32 (2016) 1353-1392.

[25] A. Iannizzotto, S. Mosconi, M. Squassina, Fine boundary regularity for the degenerate fractional $p$-Laplacian, preprint arXiv:1807.09497.

[26] A. Iannizzotto, S. Mosconi, M. Squassina, Sobolev versus Hölder minimizers for the degenerate fractional $p$-Laplacian, Nonlinear Anal. 191 (2020).

[27] S. JAROHS, Strong comparison principle for the fractional $p$-Laplacian and applications to starshaped rings, Adv. Nonlinear Stud. 18 (2018) 691-704.

[28] T. Kuusi, G. Mingione, Y. Sire, Nonlocal equations with measure data, Comm. Math. Phys. 337 (2015) $1317-1368$.

[29] T. Kuusi, G. Mingione, Y. Sire, Nonlocal self-improving properties, Anal. PDE 8 (2015) 57-114.

[30] E. Lindgren, P. Lindqvist, Fractional eigenvalues, Calc. Var. Partial Differential Equations 49 (2014) $795-826$.

[31] G. Molica Bisci, V.D. RĂdulescu, R. Servadei, Variational methods for nonlocal fractional problems, Cambridge University Press, Cambridge (2016)

[32] S. Mosconi, M. Squassina, Recent progresses in the theory of nonlinear nonlocal problems, Bruno Pini Mathematical Analysis Sem. 7 (2016) 147-164.

[33] D. Motreanu, V. V. Motreanu, N. S. Papageorgiou, A unified approach for multiple constant sign solutions and nodal solutions, Adv. Differ. Equations 12 (2007) 1363-1392.

[34] D. Motreanu, V. V. Motreanu, N. S. Papageorgiou, Topological and variational methods with applications to nonlinear boundary value problems, Springer, New York (2014).

[35] G. Palatucci, The Dirichlet problem for the p-fractional Laplace equation, Nonlinear Anal. 177 (2018) 699-732.

[36] X. Ros-Oton, J. Serra, The Dirichlet problem for the fractional Laplacian: regularity up to the boundary, J. Math. Pures Appl. 101 (2014) 275-302.

[37] X. Ros-Oton, J. Serra, The extremal solution for the fractional Laplacian, Calc. Var. Partial Differential Equations 50 (2014) $723-750$

[38] N. S. Ustinov, Multiplicity of positive solutions to the boundary-value problems for fractional Laplacians, J. Math. Sci. 236 (2019) 236-446.

[39] M. Xiang, B. Zhang, V.D. RĂdulescu, Superlinear Schrödinger-Kirchhoff type problems involving the fractional $p$ Laplacian and critical exponent, Adv. Nonlinear Anal. 9 (2020) 690-709.

[40] Z. Zhang, S. LI, On sign-changing and multiple solutions of the p-Laplacian, J. Funct. Anal. 197 (2003) $447-468$.

(S. Frassu) Department of Mathematics and Computer Science

UNIVERSITY OF CAGLIARI

Viale L. Merello 92, 09123 Cagliari, Italy

E-mail address: silvia.frassu@unica.it

(A. Iannizzotto) Department of Mathematics and Computer Science

University of CAGLiARI

Viale L. Merello 92, 09123 Cagliari, Italy

E-mail address: antonio.iannizzotto@unica.it 